\title{
Effect of Surface Roughness and Viscosity-Pressure Dependency on the Couple Stress Squeeze Film Characteristics of Parallel Circular Plates
}

\author{
Neminath Bhujappa Naduvinamani, ${ }^{1}$ Siddangouda Apparao, ${ }^{2}$ and Ayyappa G. Hiremath ${ }^{3}$ \\ ${ }^{1}$ Department of Mathematics, Gulbarga University, Gulbarga, Karnataka 585106, India \\ ${ }^{2}$ Department of Mathematics, Appa Institute of Engineering \& Technology, Gulbarga 585103, India \\ ${ }^{3}$ Department of Mathematics, Poojya Doddappa Appa College of Engineering, Gulbarga 585102, India
}

Correspondence should be addressed to Neminath Bhujappa Naduvinamani; naduvinamaninb@yahoo.co.in

Received 4 December 2013; Revised 24 March 2014; Accepted 17 April 2014; Published 25 May 2014

Academic Editor: Michel Fillon

Copyright (C) 2014 Neminath Bhujappa Naduvinamani et al. This is an open access article distributed under the Creative Commons Attribution License, which permits unrestricted use, distribution, and reproduction in any medium, provided the original work is properly cited.

\begin{abstract}
Combined effects of surface roughness and viscosity-pressure dependency on the couple stress squeeze film characteristics of parallel circular plates are presented. On the basis of Christensen's stochastic theory, two types of one-dimensional roughness structures, namely, the radial roughness and azimuthal roughness patterns, are considered and the stochastic modified Reynolds equation for these two types of roughness patterns is derived for Stokes couple stress fluid by taking into account variation of viscosity with pressure. The standard perturbation technique is employed to solve the averaged Reynolds equation and closed form expressions for the mean fluid film pressure, load carrying capacity, and squeeze film time are obtained. It is found that the effects of couple stresses and viscosity-pressure dependency are to increase the load carrying capacity, and squeeze film time for both types of roughness patterns. The effect of azimuthal (radial) roughness pattern is to increase (decrease) these squeeze film characteristics as compared to the corresponding smooth case.
\end{abstract}

\section{Introduction}

Applications of squeeze film mechanisms are observed in many areas of applied science and industrial engineering, such as machine tools, gears, bearings, rolling elements, automotive engines, and synovial joints. The squeeze film bearings with Newtonian lubricants were studied by Pinkus and Sternlicht [1], Cameron [2], and Hamrock [3]. With the development of modern machine equipment, the increasing use of fluids containing microstructures such as additives, suspensions, and long-chained polymers; for example, the length of the polymer chain may be a million times the diameter of water molecule, has received great attention in recent year. Due to this special structure of the lubricant fluid and also due to the other additives, the Newtonian fluid approximation (which neglects the size of fluid particles) is not a satisfactory engineering approach. A number of microcontinuum theories have been developed by
Ariman et al. $[4,5]$ for describing the peculiar behaviour of fluids containing substructures, which can translate, rotate, or even deform independently. Among them, the Stokes microcontinuum theory [6] is the simplest theory that allows for polar effects such as the presence of couple stresses and body couples. A number of studies have applied the Stokes microcontinuum theory to investigate the effect of couple stresses on the performance of different types of fluid-film bearings. Ramanaih [7] analyzed the squeeze film behaviour between finite plates of various shapes lubricated with couple stress fluids. Bujurke and Jayaraman [8] predicted the characteristics in a squeeze film configuration with reference to the synovial joint. Lin et al. [9] studied the pure squeeze film behaviour of long partial journal bearings with couple stress fluids under dynamic loading. The effects of couple stresses on the static and dynamic behaviour of the squeeze film lubrication of narrow porous journal bearings are analyzed by Naduvinamani et al. $[10,11]$. 
In practice, all bearing surfaces are rough to certain extent. The effect of surface roughness plays a significant role in the development of science and technology of tribology. In bearings, the height of the roughness asperities and the mean separation of the sliding surfaces are the same order. These deviations in the film thickness would be a major influencing factor in most of the bearing application. Thus, it appeared normal to view the film thickness in bearing as a stochastic process characterized by a number of statistical parameters. Christensen and Tonder $[12,13]$ derived the stochastic models for hydrodynamic lubrication of rough surface and derived generalized form of Reynold's equation applicable to rough bearings. Prakash and Tonder [14] analyzed the squeeze film between two circular plates as affected by the presence of surface asperities on the basis of Christensen's stochastic theory. The stochastic theory is more preferable than the deterministic model for the computing time and convergence problems since a large number of mesh points are required by this later model to represent the surface profile with adequate accuracy as discussed by Dowson and Jin [15]. Naduvinamani et al. [16] studied the combined effects of couple stresses and surface roughness on the performance characteristics of hydrodynamic lubrication of slider bearings with various film shapes. The combined influence of couple stresses and surface roughness on the lubrication characteristics of different journal bearings was studied by Lin et al. [17-19] and they found that the couple stress effects and the longitudinal surface roughness improve the load carrying capacity and thus decrease the attitude angle and friction parameter, while the reverse trends were observed for transverse roughness pattern.

In all these studies the effect of pressure dependency on the fluid viscosity $\mu$ is neglected and it is assumed that the viscosity $\mu$ is constant although it is a function of both pressure and temperature. According to the study by Gould [20], the variation of viscosity with pressure is important especially in the high pressure squeeze films. Lin et al. [21, 22] analyzed the theoretical study of combined effects of non-Newtonian couple stress rheology and viscosity pressure dependency (VPD) in the sphere plate squeeze film and squeeze film circular plates. Reddy et al. [23] predicted the effect of viscosity variation on the squeeze film performance of narrow hydrodynamic journal bearings with couple stress fluid.

Recently, Lin et al. [22] have studied the effects of viscosity-pressure dependency on the non-Newtonian squeeze film of parallel circular plates with an assumption that bearing surfaces are smooth. Hence the main objective of this paper is to study the effects of surface roughness on the couple stress squeeze film lubrication between circular plates by considering the pressure dependent viscosity variation which has not been studied so far.

\section{Mathematical Formulation and Solution}

Figure 1 shows the squeeze film geometry between the parallel circular plates with radius " $a$ ". The upper plate is approaching the lower plane surface (which is assumed to be

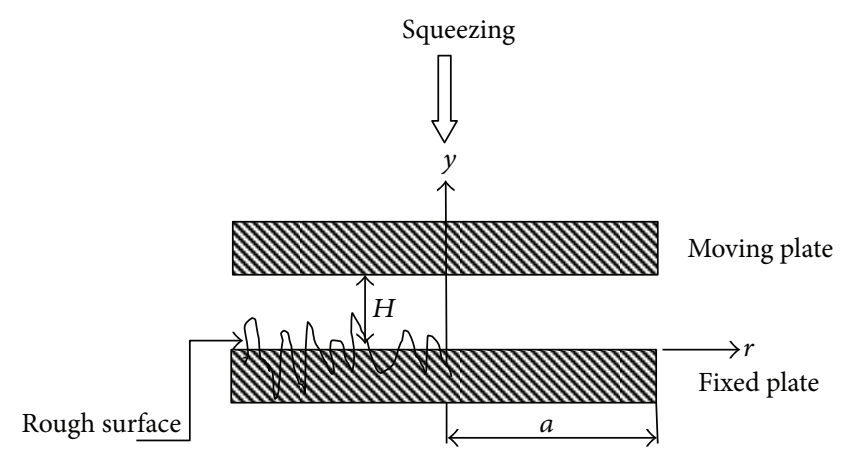

FIGURE 1: Geometry of parallel circular plates.

rough) with squeezing velocity $(-d h / d t)$ under the constant load.

The stochastic film thickness $H$ is represented by

$$
H=h+h_{s}(r, \theta, \xi),
$$

where $h$ denotes the nominal smooth part of the geometry while $h_{s}$ is the part due to the surface asperities measured from the nominal level and is randomly varying quantity of zero mean and $\xi$ is an index describing the definite roughness arrangement; hence for a given value of $\xi$, the surface roughness component $h_{s}$ of the film thickness becomes a deterministic of the space variables.

The incompressible isothermal Stokes [6] couple stress fluid is taken as the lubricant in the film region. It is assumed that the body forces, body couples, and fluid inertia are negligible, but the viscosity $\mu$ varies with squeeze film pressure. Using thin film assumption, the basic equations governing the lubricant velocity and pressure reduce to

$$
\begin{gathered}
\frac{1}{r} \frac{\partial}{\partial r}(r u)+\frac{\partial v}{\partial y}=0 \\
\frac{\partial}{\partial y}\left(\mu \frac{\partial u}{\partial y}\right)-\eta \frac{\partial^{4} u}{\partial y^{4}}=\frac{\partial p}{\partial r} \\
\frac{\partial p}{\partial y}=0,
\end{gathered}
$$

where $u$ and $v$ are the velocity components in the $r$ and $y$ direction, respectively, $p$ is the pressure, $\mu$ is the dynamic viscosity, and $\eta$ represents a new material constant responsible for the couple stress fluids. To take into account the variation of viscosity with pressure dependency of lubricant the formula proposed by Barus [24] is considered:

$$
\mu=\mu_{0} \exp (\beta p),
$$

where $\beta$ denotes the coefficient of viscosity pressure dependency (VPD) and $\mu_{0}$ is the viscosity at ambient pressure and a constant temperature. The lubricant viscosity is increasing approximately exponentially; it could therefore alter the predicted performance of squeeze film bearings. 
The boundary conditions for velocity components $u(r, y)$ and $v(r, y)$ at the surface of plates are

$$
\begin{array}{cc}
u(r, 0)=\frac{\partial^{2} u(r, 0)}{\partial y^{2}}=0 ; & v(r, 0)=0 ; \\
u(r, H)=\frac{\partial^{2} u(r, H)}{\partial y^{2}}=0 ; & v(r, H)=-\frac{d h}{d t} .
\end{array}
$$

Solving (3) and using relation (5) with boundary conditions (6) yield

$$
\begin{aligned}
u(r, y)= & \frac{\exp (-2 \beta p)}{2 \mu_{0}} \frac{\partial p}{\partial r} \\
& \times\left\{\frac{y^{2}-y H}{\exp (-\beta p)}+2 l^{2}\right. \\
& \left.\quad-\frac{2 l^{2} \cosh [(2 y-H) / \exp (-0.5 \beta p) 2 l]}{\cosh [H / \exp (-0.5 \beta p) 2 l]}\right\},
\end{aligned}
$$

$$
\text { where } l=\sqrt{\frac{\eta}{\mu_{0}}} \text { is couple stress parameter. }
$$

The non-Newtonian couple stress Reynold's type equation for the squeeze film pressure can be obtained by substituting the expression for $u$ obtained in (7) into the continuity equation (2) and integrating across the film thickness using the boundary conditions of $v$ given in (6) in the form

$$
\frac{1}{r} \frac{\partial}{\partial r}\left\{\varphi(H, l, \beta, p) r \frac{\partial p}{\partial r}\right\}=12 \mu_{0} \frac{d H}{d t},
$$

where

$$
\begin{aligned}
\varphi(H, l, \beta, p)= & H^{3} \exp (-\beta p)-12 l^{2} H \exp (-2 \beta p) \\
& +24 l^{3} \exp (-2.5 \beta p) \tanh [\exp (0.5 \beta p) H / 2 l] .
\end{aligned}
$$

Let $f\left(h_{s}\right)$ be the probability density function of the stochastic film thickness $h_{s}$. Taking the stochastic average of (9) with respect to $f\left(h_{s}\right)$ the averaged modified Reynolds type equation is obtained in the form

$$
\frac{1}{r} \frac{\partial}{\partial r}\left\{E[\varphi(H, l, \beta, p)] r \frac{\partial E[p]}{\partial r}\right\}=12 \mu_{0} \frac{d E[H]}{d t},
$$

where the expectancy operator $E(\cdot)$ is defined by

$$
E(\cdot)=\int_{-\infty}^{\infty}(\cdot) f\left(h_{s}\right) d h_{s} .
$$

Since most of the engineering rough surfaces are Gaussian in nature, a polynomial form is chosen to approximate the Gaussian distribution by

$$
f\left(h_{s}\right)= \begin{cases}\frac{35}{32 c^{7}}\left(c^{2}-h_{s}^{2}\right)^{3} ; & -c<h_{s}<c \\ 0 & \text { otherwise, }\end{cases}
$$

where $c$ is the half total range of random film thickness variable. The function terminates at $c= \pm 3 \sigma$ where $\sigma$ is the standard deviation.

Introducing the nondimensional variables and parameters

$$
\begin{array}{lll}
r^{*}=\frac{r}{a}, & p^{*}=\frac{E(p) h_{0}^{3}}{\mu_{0} a^{2}(-d h / d t)}, & H^{*}=\frac{H}{h_{0}}, \\
K=\frac{l}{h_{0}}, & V=\frac{\beta \mu_{0} a^{2}(-d h / d t)}{h_{0}^{3}}, & c^{*}=\frac{c}{h_{0}},
\end{array}
$$

where $h_{0}$ is minimum film thickness.

Into (11), the nondimensional non-Newtonian couple stress Reynold's type equation is obtained in the form

$$
\frac{\partial}{\partial r^{*}}\left\{E\left[\varphi^{*}\left(H^{*}, V, K, p^{*}\right) r^{*} \frac{\partial p^{*}}{\partial r^{*}}\right]\right\}=-12 r^{*},
$$

where

$$
\begin{aligned}
\varphi^{*}\left(H^{*}, V, K, p^{*}\right)= & H^{* 3} \exp \left(-V p^{*}\right) \\
& -12 K^{2} H^{*} \exp \left(-2 V p^{*}\right) \\
& +24 K^{3} \exp \left(-2.5 V p^{*}\right) \\
& \times \tanh \left[\exp \left(0.5 V p^{*}\right) \frac{H^{*}}{2 K}\right] .
\end{aligned}
$$

In accordance with the Christensen [12] stochastic theory, the analysis is done for two types of one-dimensional surface roughness patterns, namely, one-dimensional radial roughness pattern and azimuthal roughness pattern.

For the one-dimensional radial roughness pattern, the roughness striations in the form of ridges and valleys running in the $r$-direction, in this case the nondimensional film thickness assumes the form

$$
H^{*}=h^{*}+h_{s}^{*}(\theta, \xi) .
$$

For the one-dimensional azimuthal roughness pattern, the roughness striations in the form of ridges and valleys running in the $\theta$-direction, in this case the nondimensional film thickness assumes the form

$$
H^{*}=h^{*}+h_{s}^{*}(r, \xi) .
$$

Then the modified stochastic Reynold's type Equation (15) for these two types of roughness pattern takes the form

$$
\frac{\partial}{\partial r^{*}}\left\{G^{*}\left(H^{*}, V, K, p^{*}, c^{*}\right) r^{*} \frac{\partial p^{*}}{\partial r^{*}}\right\}=-12 r^{*},
$$

where

$$
\begin{aligned}
G^{*} & \left(H^{*}, V, K, p^{*}, c^{*}\right) \\
& = \begin{cases}E\left[\varphi^{*}\left(H^{*}, V, K, p^{*}\right)\right] & \text { for radial roughness } \\
E\left[\frac{1}{\varphi^{*}\left(H^{*}, V, K, p^{*}\right)}\right]^{-1} & \text { for azimuthal roughness. }\end{cases}
\end{aligned}
$$


The nondimensional Reynold's Equation (19) is observed to be highly nonlinear. To obtain the first order analytical solution for small values of the viscosity parameter $0 \leq$ $V \ll 1$, a small perturbation method for the film pressure is adopted following the procedure by Gould [20]

$$
p^{*}=p_{0}^{*}+V p_{1}^{*}
$$

into the Reynold's type equation and neglecting second and higher order of $V$, we get the following two equations responsible for pressures $p_{0}^{*}$ and $p_{1}^{*}$

$$
\begin{gathered}
\frac{\partial}{\partial r^{*}}\left\{r^{*} \frac{\partial p_{0}^{*}}{\partial r^{*}}\right\}=-\frac{12 r^{*}}{G_{0}^{*}\left(H^{*}, K, c^{*}\right)}, \\
\frac{\partial}{\partial r^{*}}\left\{r^{*} \frac{\partial p_{1}^{*}}{\partial r^{*}}\right\}=-\frac{G_{1}^{*}\left(H^{*}, K, c^{*}\right)}{G_{0}^{*}\left(H^{*}, K, c^{*}\right)} \frac{\partial}{\partial r^{*}}\left\{p_{0}^{*} r^{*} \frac{\partial p_{0}^{*}}{\partial r^{*}}\right\},
\end{gathered}
$$

where

$$
\begin{aligned}
\varphi_{0}^{*}\left(H^{*}, K\right)= & H^{* 3}-12 K^{2} H^{*}+24 K^{3} \tanh \left[\frac{H^{*}}{2 K}\right], \\
\varphi_{1}^{*}\left(H^{*}, K\right)= & -H^{* 3}+6 K^{2} H^{*}\left\{4+\sec ^{2} h\left[\frac{H^{*}}{2 K}\right]\right\} \\
& -60 K^{3} \tanh \left[\frac{H^{*}}{2 K}\right] .
\end{aligned}
$$

Solving (22) under the pressure boundary conditions for circular squeeze film plates is

$$
\begin{gathered}
\frac{d p_{0}^{*}}{d r^{*}}=\frac{d p_{1}^{*}}{d r^{*}}=0 \quad \text { at } r^{*}=0 \\
p_{0}^{*}=p_{1}^{*}=0 \quad \text { at } r^{*}=1 .
\end{gathered}
$$

The dimensionless pressure developed in the film region is

$$
\begin{aligned}
p^{*}= & \frac{3\left(1-r^{* 2}\right)}{G_{0}^{*}\left(H^{*}, K, c^{*}\right)}-\frac{9}{2} V \frac{G_{1}^{*}\left(H^{*}, K, c^{*}\right)}{\left\{G_{0}^{*}\left(H^{*}, K, c^{*}\right)\right\}^{3}} \\
& \times\left(1-2 r^{* 2}+r^{* 4}\right) .
\end{aligned}
$$

The load carrying capacity can be obtained by integrating the film pressure over the squeezing film area as follows:

$$
E(W)=\int_{r=0}^{a} E(p) 2 \pi r d r
$$

This takes the dimensionless form

$$
W^{*}=\frac{3 \pi}{2} \frac{\left\{G_{0}^{*}\left(H^{*}, K, c^{*}\right)\right\}^{2}-V G_{1}^{*}\left(H^{*}, K, c^{*}\right)}{\left\{G_{0}^{*}\left(H^{*}, K, c^{*}\right)\right\}^{3}},
$$

where

$$
W^{*}=\frac{E(W) h_{0}^{3}}{\mu_{0} a^{4}(-d h / d t)}
$$

The squeezing time can be calculated by integrating (27) with respect to $h^{*}$ under the condition $h^{*}=1$ at $t^{*}=0$ as follows:

$$
t^{*}=\frac{3 \pi}{2} \int_{h^{*}}^{1} \frac{\left\{G_{0}^{*}\left(H^{*}, K, c^{*}\right)\right\}^{2}-V G_{1}^{*}\left(H^{*}, K, c^{*}\right)}{\left\{G_{0}^{*}\left(H^{*}, K, c^{*}\right)\right\}^{3}} d h^{*},
$$

where

$$
t^{*}=\frac{E(W) h_{0}^{2}}{\mu_{0} a^{4}} t .
$$

It is noted that the values of the dimensionless film pressure, the dimensionless load carrying capacity, and the dimensionless time cannot be obtained by direct integration; however, they could be numerically evaluated by the Gaussian quadrature method.

\section{Results and Discussions}

The effects of surface roughness and viscosity pressure dependency on the couple stress squeeze film between parallel circular plates in the presence of azimuthal roughness pattern and the radial roughness pattern are analyzed. The effect of couple stresses resulting from the addition of various additives to the Newtonian lubricant is identified by the couple stress parameter $K$. On the basis of the Stokes couple stress fluid theory, the new material constant $\eta$ appearing in (3) is responsible for the non-Newtonian property of couple stresses. The value of this new material constant $\eta$ could be determined by some experiments. For example, the lubricant viscosity $\mu$ can be determined experimentally from Couette flow with zero pressure gradient; after knowing $\mu$, one can obtain the flow rate $Q_{0}$ of Poiseuille flow with no couple stresses for a given gap and pressure gradient; since the flow rate $Q$ of Poiseuille flow with couple stresses can be obtained by actual experiment and can be displayed by $Q=$ $Q$ (gap, $\mu, \eta$ ), the value of $\eta$ can then be determined. Further details are discussed by Stokes [6].

From the Barus formula for isothermal viscosity pressure dependency of lubricants, the effect of variation of viscosity with pressure is shown by viscosity parameter $V$. The roughness parameter $c^{*}$ signifies the effect of surface roughness resulting from the large vertical deviations of a real surface. The limiting case $c^{*} \rightarrow 0$ is the circular squeeze film plates under the viscous, non-Newtonian couple stress fluids corresponding to smooth case studied by Lu and Lin [21]. In the present study squeeze film characteristics are presented with $V=0.05$, couple stress parameters within the range of $K=0.0 \sim 0.2$, and roughness parameter within the range $c^{*}=$ $0.0 \sim 0.3$.

3.1. Squeeze Film Pressure. The variation of nondimensional mean pressure $p^{*}$ as a function of nondimensional radial coordinate $r^{*}$ for different values of $V$ and $K$ is depicted in Figure 2 with the parametric values of $h^{*}=0.4$ and $c^{*}=$ 0.2 for both types of roughness patterns. It is observed that the effect of isoviscous (IV) non-Newtonian lubrication (IV, $K=0.1)$ is to increase $p^{*}$ in either case as compared to 


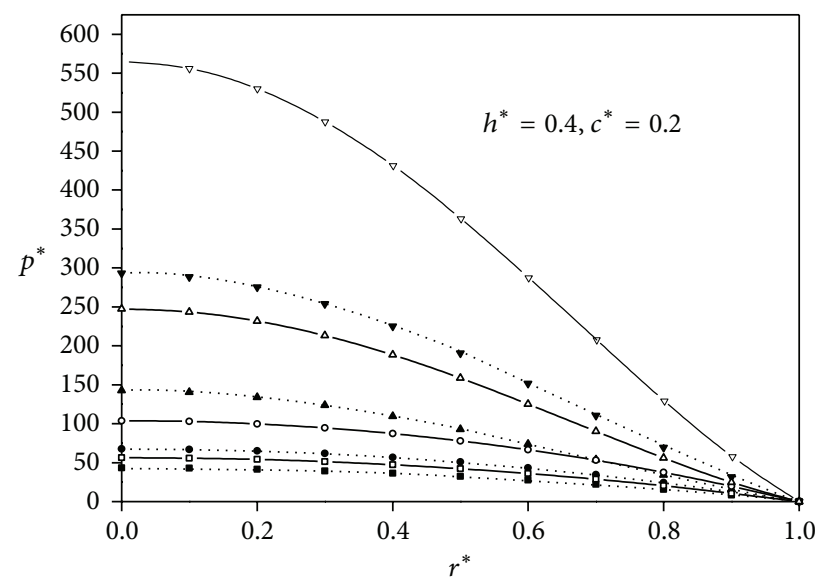

$$
\begin{aligned}
& \text { Radial } \\
& \text {-.. IV }=0, K=0 \text { (Newt) } \\
& \text {-.. IV }=0, K=0.1 \\
& \text { ^. V }=0.05, K=0.1 \\
& \text { 、. V }=0.05, K=0.2 \\
& \text {-ם- IV }=0, K=0 \text { (Newt) } \\
& \text {-०- IV }=0, K=0.1 \\
& \text { - } \Delta-\mathrm{V}=0.05, K=0.1 \\
& \text { - }-\mathrm{V}=0.05, K=0.2
\end{aligned}
$$

FIGURE 2: Film pressure $p^{*}$ versus radial coordinate $r^{*}$ for different $V$ and $K$.

isoviscous Newtonian case. It is also observed that the effect of viscosity pressure dependency and the non-Newtonian lubrication $(V=0.05, K=0.1,0.2)$ is to increase $p^{*}$ in either case as compared to isoviscous non-Newtonian lubrication. Further, the increase in $p^{*}$ is more pronounced for the azimuthal roughness pattern as compared to the radial roughness pattern. This is because, in the case of azimuthal roughness pattern, the roughness striations are in the form of ridges and valleys running in the $\theta$-direction which blocks the flow of lubricant, while in the case of the radial roughness pattern, the roughness striations are in the form of ridges and valleys running in the $r$-direction by which the lubricant can escape easily.

Figure 3 shows the variation of $p^{*}$ as a function of $r^{*}$ for different values of roughness parameter $c^{*}$ with $V=0.05$, $K=0.1$, and $h^{*}=0.4$ for both types of roughness pattern. It is found that $p^{*}$ increases (decreases) for azimuthal (radial) roughness pattern.

3.2. Load Carrying Capacity. The variation of nondimensional load carrying capacity $W^{*}$ with film thickness $h^{*}$ for different values of $V$ and $K$ with roughness parameter $c^{*}=0.2$ is plotted in Figure 4 for both types of roughness patterns. It is observed that the effect of isoviscous nonNewtonian couple stress lubricants is to increase $W^{*}$ in either case as compared to isoviscous Newtonian case. It is also observed that the effect of viscosity pressure dependency and the couple stress fluid lubrication is to increase $W^{*}$ in both cases as compared to isoviscous non-Newtonian case. Further, increase in $W^{*}$ is more accentuated for the azimuthal roughness pattern as compared to the radial roughness pattern. Figure 5 shows the variation of $W^{*}$ as a function of $h^{*}$ with $V=0.05$ and $K=0.1$ for different values of roughness parameter $c^{*}$ for both types of roughness patterns.

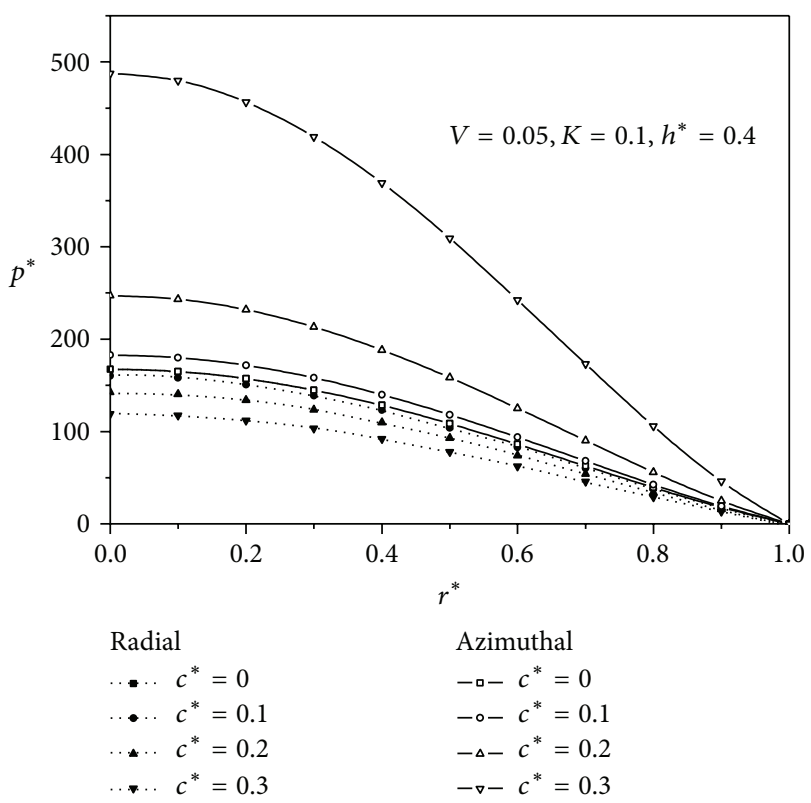

FIGURE 3: Film pressure $p^{*}$ versus radial coordinate $r^{*}$ for different $c^{*}$.

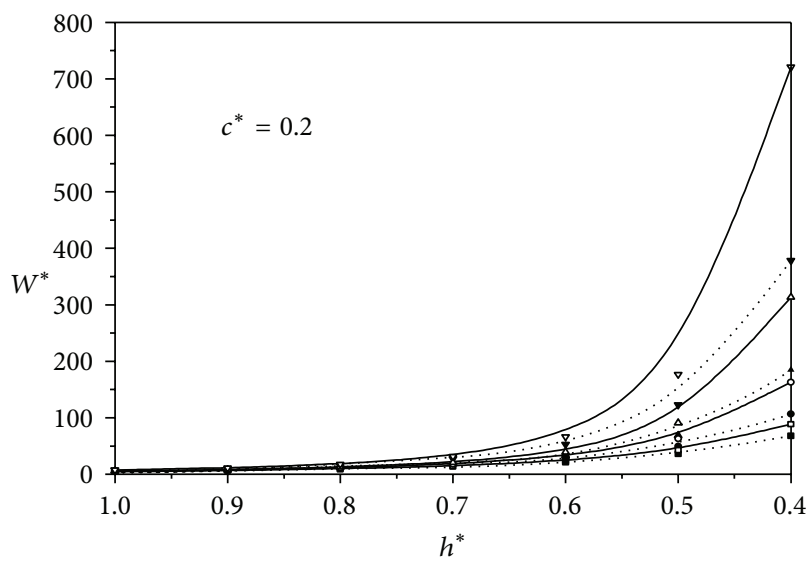

$$
\begin{aligned}
& \text { Radial Azimuthal } \\
& \text { - } \cdots \mathrm{IV}=0, K=0 \text { (Newt.) } \quad \text { - - } \mathrm{IV}=0, K=0 \text { (Newt.) } \\
& \bullet \text { IV }=0, K=0.1 \quad \text { - o- } \text { IV }=0, K=0.1 \\
& \text {.. } \mathrm{V}=0.05, K=0.1 \quad-\Delta-\mathrm{V}=0.05, K=0.1 \\
& \text {-. } \mathrm{V}=0.05, K=0.2 \quad-\nabla-\mathrm{V}=0.05, K=0.2
\end{aligned}
$$

FIGURE 4: Load capacity $w^{*}$ versus the film thickness $h^{*}$ for different $V$ and $K$.

It is interesting to note that the effect of azimuthal (radial) roughness patterns is to increase (decrease) $W^{*}$ as compared to the corresponding to smooth case $\left(c^{*}=0\right)$. At $c^{*}=0.3$ (i.e., with the vertical deviations being large) it is observed that the increase (decrease) in $W^{*}$ is more pronounced for azimuthal (radial) roughness pattern. The large amount of load is delivered for azimuthal roughness pattern as compared to the radial roughness pattern.

3.3. Squeeze Film Time. Figure 6 shows the variation of dimensionless squeeze film time $t^{*}$ with the non-Newtonian 


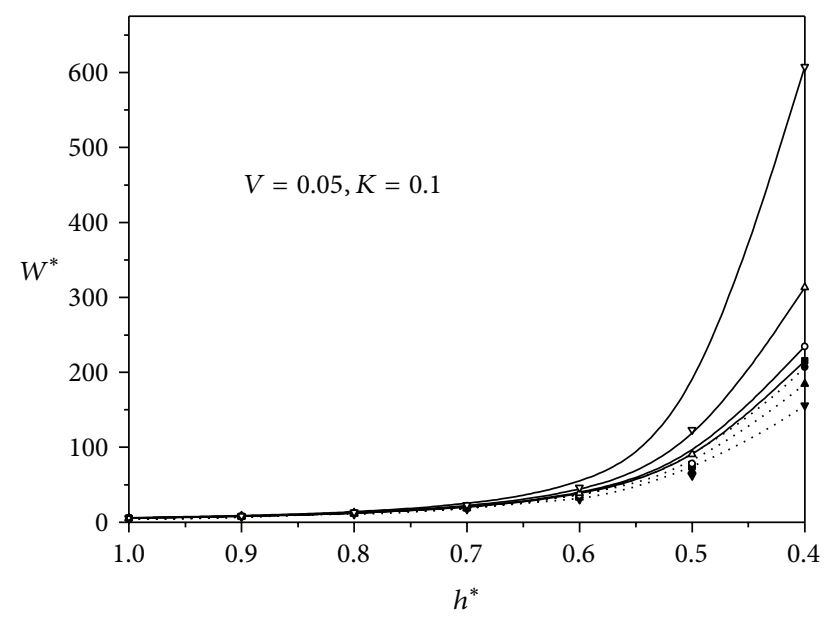

$\begin{array}{ll}\text { Radial } & \text { Azimuthal } \\ \cdots \cdots c^{*}=0.0 & -\square-c^{*}=0.0 \\ \cdots \cdots c^{*}=0.1 & -\triangleright-c^{*}=0.1 \\ \cdots \cdots c^{*}=0.2 & -\Delta-c^{*}=0.2 \\ \cdots \cdots c^{*}=0.3 & \end{array}$

FIGURE 5: Load capacity $w^{*}$ versus the film thickness $h^{*}$ for different $c^{*}$.

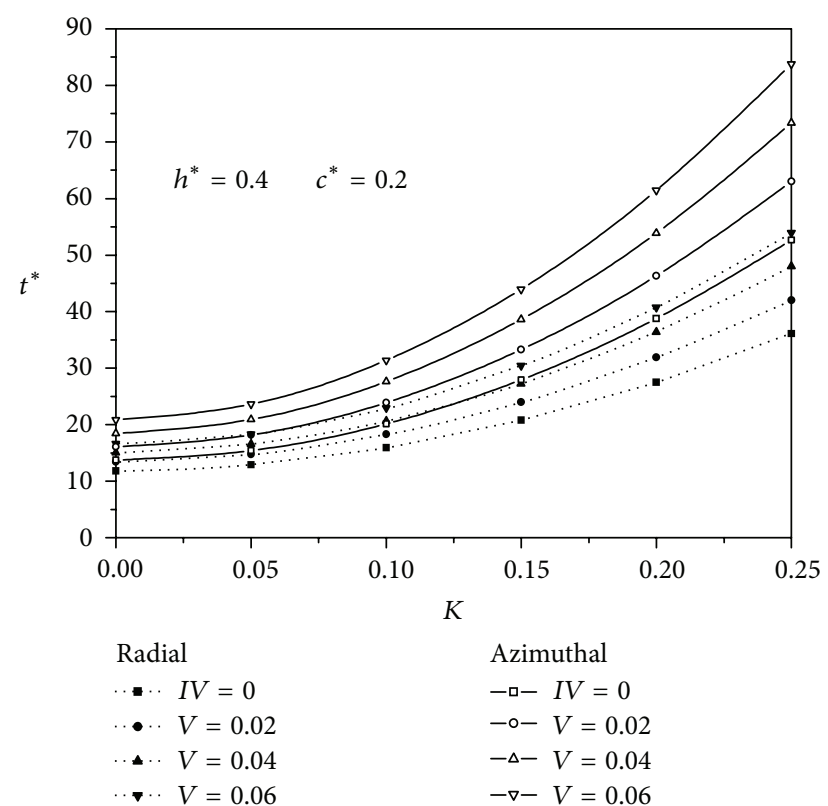

Figure 6: Dimensionless time $t^{*}$ versus non-Newtonian parameter $K$ for different $V$.

parameter $K$ with $h^{*}=0.4$ and $c^{*}=0.2$ for different values of $V$ for both types of roughness patterns. It is interesting to note that the effect of viscosity pressure dependency is to increase $t^{*}$ in either case as compared to the isoviscous case. Further, the increase (decrease) in $t^{*}$ is more for the azimuthal (radial) roughness patterns. Figure 7 shows the effect of roughness parameters $c^{*}$ on the variation of $t^{*}$ with $K$ for both types of roughness patterns. It is found that due to the presence

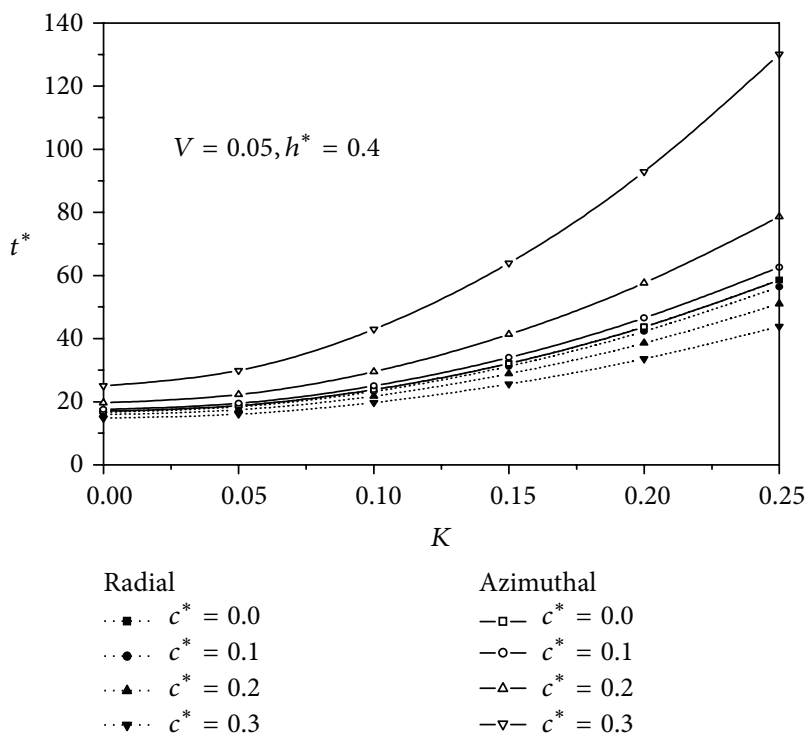

Figure 7: Dimensionless time $t^{*}$ versus non-Newtonian parameter $K$ for different $c^{*}$.

of surface roughness, as the height of the vertical deviations of surface increases the response time $t^{*}$ also increases. This increase is more for azimuthal roughness patterns than the radial roughness pattern.

\section{Conclusions}

The effect of surface roughness and viscosity pressure dependency on the couple stress squeeze film characteristics of parallel circular plates is analyzed on the basis of Reddy et al. [23] experimental formula and Christensen [12] stochastic theory for rough surfaces. According to the results discussed, the following conclusions can be drawn.

(1) The effect of viscosity variation parameter is to enhance the load carrying capacity and response time as compared to the isoviscous case.

(2) The effect of non-Newtonian couple stress fluid is to increase the load carrying capacity and to lengthen the squeeze film time as compared to the corresponding Newtonian case.

(3) The presence of azimuthal surface roughness pattern on the bearing surface improves the squeeze film characteristics whereas the performance of the squeeze film suffers due to the presence of radial roughness pattern.

\section{Conflict of Interests}

The authors declare that there is no conflict of interests regarding the publication of this paper. 


\section{References}

[1] O. Pinkus and B. Sternlicht, Theory of Hydrodynamic Lubrication, McGraw-Hill, New York, NY, USA, 1961.

[2] A. Cameron, The Principles of Lubrication, Longmans, Green and Company, London, UK, 1966.

[3] B. J. Hamrock, Fundamentals of Fluid Film Lubrication, McGraw-Hill, New York, NY, USA, 1994.

[4] T. Ariman, M. A. Turk, and N. D. Sylvester, "Microcontinuum fluid mechanics-a review," International Journal of Engineering Science, vol. 11, no. 8, pp. 905-930, 1973.

[5] T. Ariman, M. A. Turk, and N. D. Sylvester, "Applications of microcontinuum fluid mechanics," International Journal of Engineering Science, vol. 12, no. 4, pp. 273-293, 1974.

[6] V. K. Stokes, "Couple stresses in fluids," Physics of Fluids, vol. 9, pp. 1709-1715, 1966.

[7] G. Ramanaiah, "Squeeze films between finite plates lubricated by fluids with couple stress," Wear, vol. 54, no. 2, pp. 315-320, 1979.

[8] N. M. Bujurke and G. Jayaraman, "The influence of couple stresses in squeeze films," International Journal of Mechanical Sciences, vol. 24, no. 6, pp. 369-376, 1982.

[9] J. R. Lin, Y. Pun, Y. Kang, and K. C. Cha, "Pure squeeze film behavior of long journal bearings with couple stress fluids under dynamic loading," Transactions of the Canadian Society for Mechanical Engineering, vol. 24, no. 3-4, pp. 477-485, 2000.

[10] N. B. Naduvinamani, P. S. Hiremath, and G. Gurubasavaraj, "Static and dynamic behaviour of squeeze-film lubrication of narrow porous journal bearings with coupled stress fluid," Proceedings of the Institution of Mechanical Engineers, Part J: Journal of Engineering Tribology, vol. 215, no. 1, pp. 45-62, 2001.

[11] N. B. Naduvinamani, P. S. Hiremath, and G. Gurubasavaraj, "Squeeze film lubrication of a short porous journal bearing with couple stress fluids," Tribology International, vol. 34, no. 11, pp. 739-747, 2001.

[12] H. Christensen, "Stochastic models for hydrodynamic lubrication of rough surfaces," Proceedings of the Institution of Mechanical Engineers, vol. 184, pp. 1013-1026, 1969.

[13] H. Christensen and K. Tonder, "The hydrodynamic lubrications of rough journal bearings," Journal of Lubrication Technology, vol. 95, no. 2, pp. 166-172, 1973.

[14] J. Prakash and K. Tonder, "Roughness effects in circular squeeze plates," ASLE Transactions, vol. 20, no. 3, pp. 257-263, 1977.

[15] D. Dowson and Z. M. Jin, "Micro-elastohydrodynamic lubrication of low elastic modulus solids on rigid substrates," Journal of Physics D: Applied Physics, vol. 25, no. 1A, pp. A116-A123, 1992.

[16] N. B. Naduvinamani, S. T. Fathima, and P. S. Hiremath, "Hydrodynamic lubrication of rough slider bearings with couple stress fluids," Tribology International, vol. 36, no. 12, pp. 949-959, 2003.

[17] J. R. Lin, C. H. Hsiu, and H. L. Chiang, "Combined effects of couple stresses and surface roughness on the lubrication of short journal bearings," Industrial Lubrication and Tribology, vol. 55, no. 5, pp. 233-243, 2003.

[18] J. R. Lin, L. C. Hsiu, and C. H. Hsu, "Lubrication performance of finite journal bearings considering effects of couple stresses and surface roughness," Tribology International, vol. 37, no. 4, pp. 297-307, 2004.

[19] J. R. Lin, H. L. Chiang, C. H. Hsu, and Y. P. Chang, "Linear stability analysis of a rough short journal bearing lubricated with non-Newtonian fluids," Tribology Letters, vol. 17, no. 4, pp. 867-877, 2004.
[20] P. Gould, "High pressure spherical squeeze films," Journal of Lubrication Technology, vol. 93, no. 1, pp. 207-208, 1971.

[21] R. F. Lu and J. R. Lin, "A theoretical study of combined effects of non-Newtonian rheology and viscosity-pressure dependence in the sphere-plate squeeze-film system," Tribology International, vol. 40, no. 1, pp. 125-131, 2007.

[22] J. R. Lin, L. M. Chu, and L. J. Liang, "Effects of viscosity pressure dependency on the non-Newtonian squeeze film of parallel circular plate," Lubrication Science, vol. 25, no. 1, pp. 1-9, 2013.

[23] G. J. C. Reddy, C. E. Reddy, and K. R. K. Prasad, "Effects of viscosity variation on the squeeze film performance of narrow hydrodynamic journal bearing operating with couple stress fluids," Proceedings of the Institution of Mechanical Engineers, Part J: Journal of Engineering Tribology, vol. 222, no. 2, pp. 141150, 2000.

[24] C. Barus, "Isothermals, isopiestics and isometric relative to viscosity," The American Journal of Science, vol. 45, pp. 87-96, 1893. 

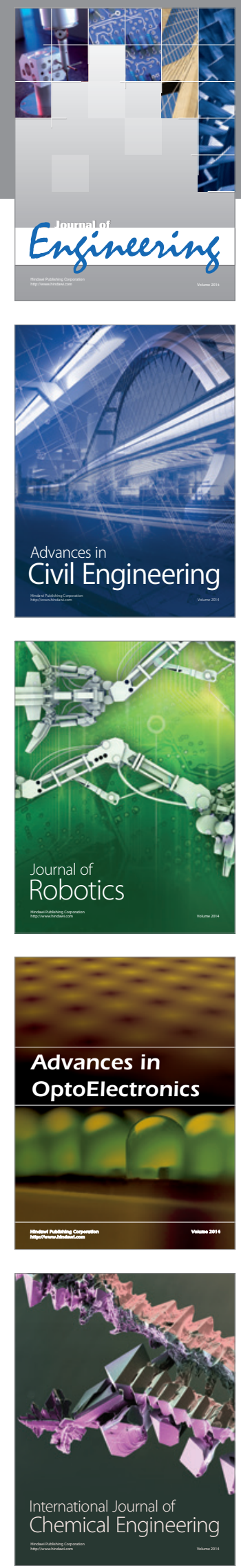

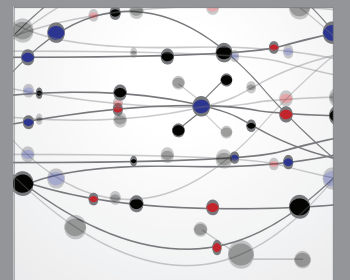

The Scientific World Journal
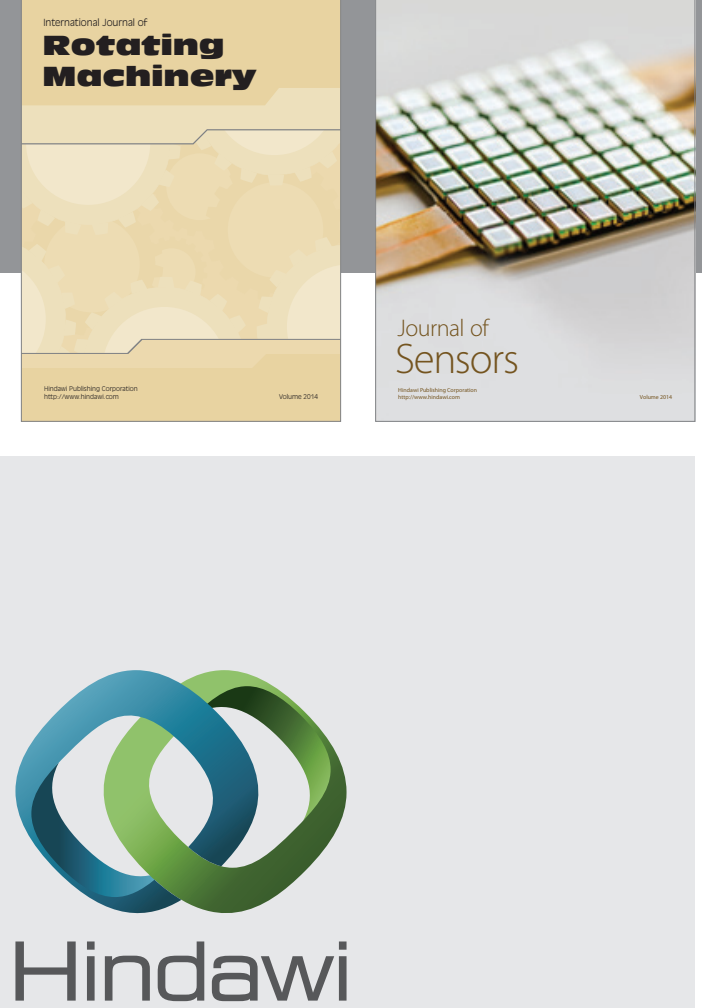

Submit your manuscripts at http://www.hindawi.com
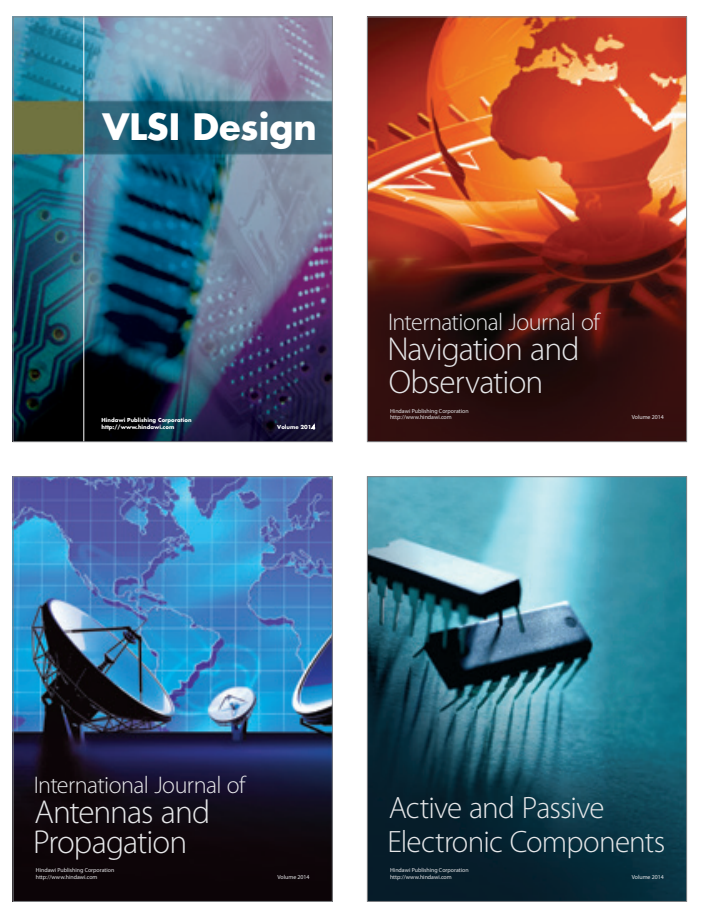
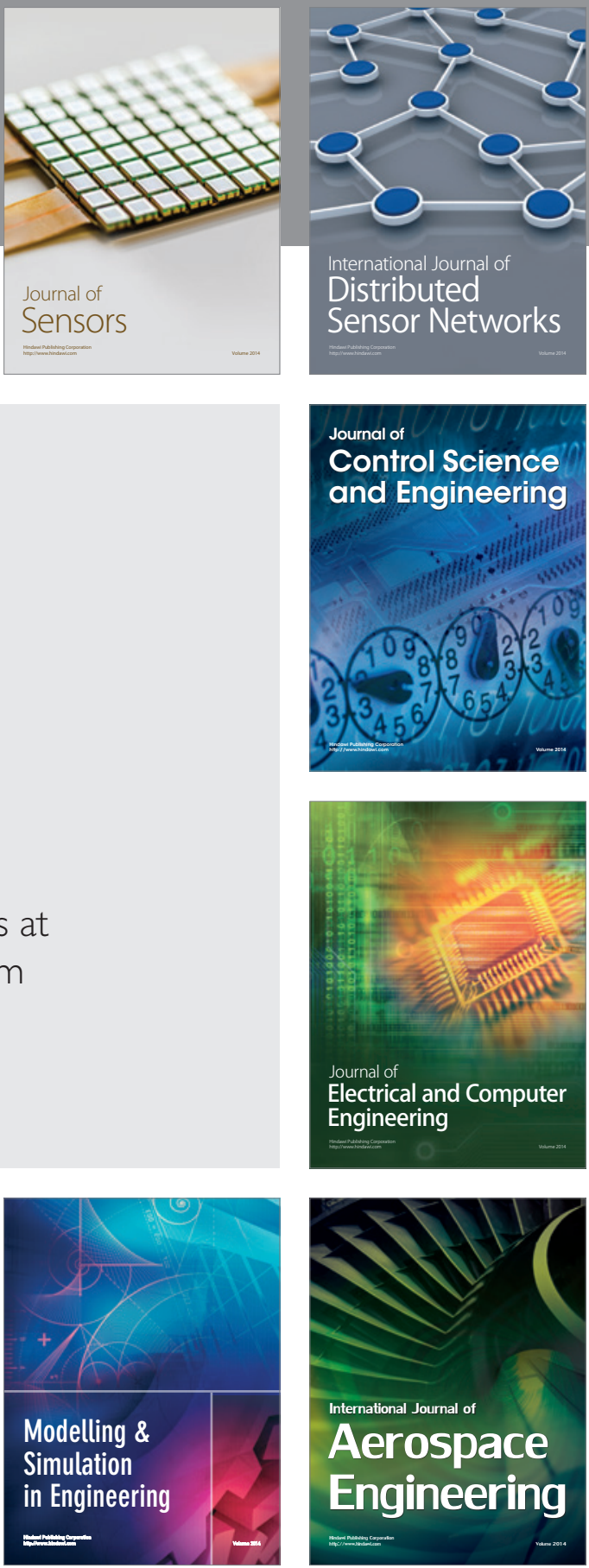

Journal of

Control Science

and Engineering
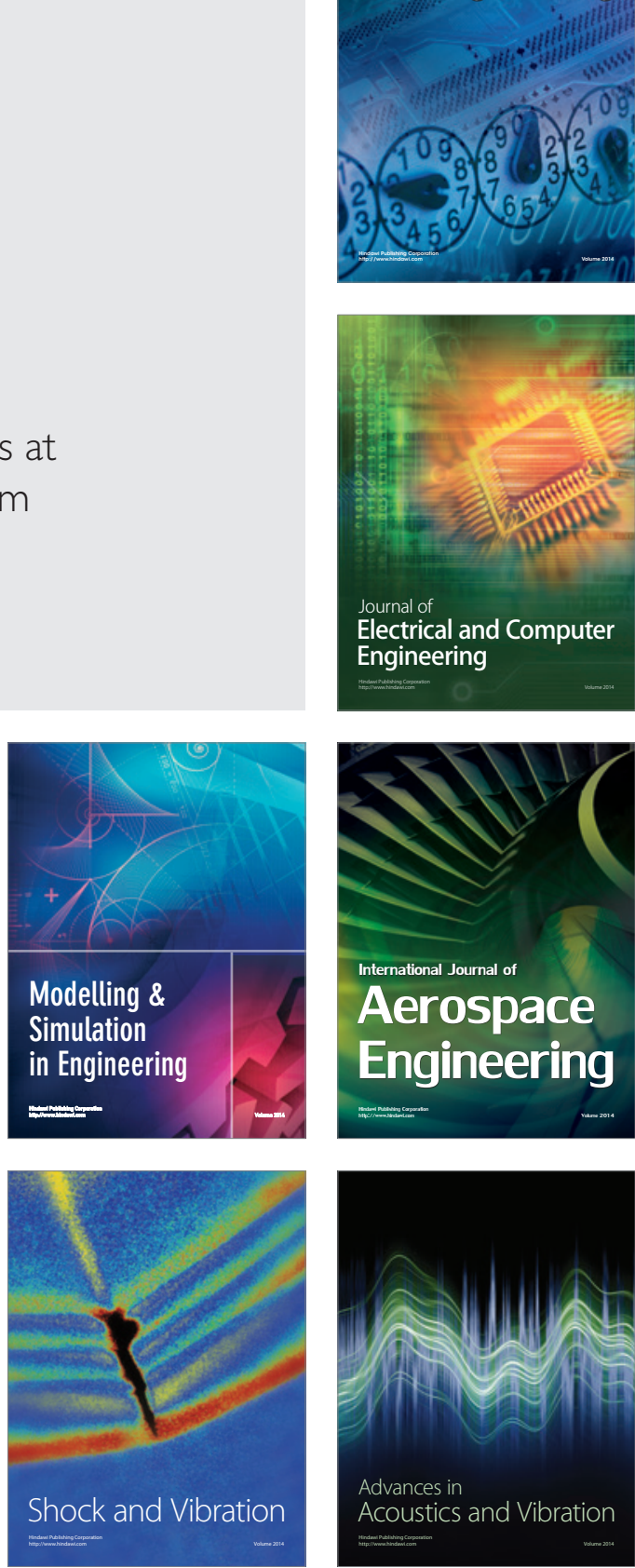